\title{
LA TRADUCTION ET LES LIMITES DE LA FIDÉLITÉ
}

Abstract. Bogacki Krzysztof, La traduction et les limites de la fidélité [Translation and the limits of fidelity]. Studia Romanica Posnaniensia. Adam Mickiewicz University Press, Poznań, vol. XXV/XXVI: 2000, pp. 29-40. ISBN 83-232-0965-0. ISSN 0137-2475.

This article is devoted to the problem of divergence occurring among different versions of translation of the same text. On the basis of investigated translations by Polish oustanding poets of La Fontaine's Fables into Polish the author offers a classification of divergences found. This classification takes into consideration both the semantic and formal factors.

\section{INTRODUCTION}

Si les modèles de l'activité traduisante diffèrent entre eux, ils concordent tous sur un point: ils tiennent compte, d'une manière ou d'une autre, de l'exigence de rester fidèle à l'original ${ }^{1}$. Cette idée préoccupe non seulement les professionnels: linguistes, traducteurs et interprètes. Elle est profondément encrée dans la langue de tous les jours à tel point qu'elle est même devenue partie intégrante d'expressions diverses employées au sens non-technique. Ainsi on parle volontiers de traduction fidèle, on demande à l'élève ou à l'interprète de rester fidèle à l'original etc. La même préoccupation de fidélité se cache derrière les expressions telles que traduction libre vs traduction fidèle etc. qui, elles, font partie des termes techniques utilisés en traductologie ${ }^{2}$. Un modèle de fidélité en traduction est proposé par A. Hurtado-Albir (1990). Trois éléments indissociables y apparaissent: le vouloir dire de l'auteur, la langue d'arrivée, le lecteur. Selon l'auteur, trahir un seul revient à manquer à l'exigence fondamentale de fidélité.

'Il existe différents modèles théoriques qui décrivent la situation dans laquelle se trouve celui à qui incombe la tâche de traduire un texte d'une langue dans une autre. Les noms que l'on pourrait citer ici sont ceux de J. Catford, D. Seleskovitch, M. Lederer, F. Grucza, J. Łukszyn, E. Nida et d'autres.

${ }^{2}$ Cf. J. Łukszyn (1993), A. Pisarska, T. Tomaszkiewicz (1996). 
Restant à un degré de généralité assez élevé, les théoriciens de la traduction mettent la notion de fidélité en avant, tous l'affirment haut et fort, tout en sachant pertinamment que la fidélité reste une notion ambiguë. L'ambiguité inhérente à cette notion reste incontournable même si on essaie de l'étudier par le biais du concept d'équivalence. De telles tentatives ont toutes conduit à en proposer plusieurs variantes. On les trouve sous la plume des chercheurs venus d'horizons divers. Ainsi M. Lederer (1994) distingue entre équivalence cognitive et équivalence affective; on souligne aussi que la traduction consiste non seulement en une mise en relation des sens préétablis liés à des fragments du texte: traduire c'est aussi restaurer au maximum dans la langue-cible le réseau des associations et de valeurs de toute sorte véhiculées par l'original. E. Nida $(1964,1975)$ parle d'équivalence formelle lorsqu'une catégorie grammaticale dans la langue-source est traduite par la catégorie identique dans la langue-cible. C'est cependant l'école allemande de traduction qui a insisté le plus sur cette notion. Ainsi W. Koller (1979) distingue entre équivalence de connotation, équivalence référentielle, normative, pragmatique, formelle et esthétique. D'autres auteurs font des distinctions différentes ce qui aboutit à un tableau global assez complexe ${ }^{3}$.

La pratique quotidienne montre que, très souvent, l'original et la traduction sont séparés par des écarts que l'on juge intuitivement comme plus ou moins importants. Ceux-ci sont-ils plus dangereux que ceux-là ? Peut-on quantifier la distance qui sépare l'original de la traduction? Ou sommes-nous condamnés, au contraire, à des appréciations entachés de subjectivité? Dans ce qui suit, nous allons essayer de montrer que la fidélité à l'original dans la traduction a plus d'un visage. D'un autre côté, nous tâcherons de voir quelles sont les difficultés auxquelles on se heurte si l'on essaie de dépasser le seuil des constatations intuitives et d'aller vers des formulations plus précises, quantifiables si possible.

\section{LES TYPES D'ÉCARTS}

Si l'on élimine d'emblée les cas d'erreurs manifestes - ceux-ci font la joie des lapsologues dont le corpus est constamment alimenté non seulement par les copies d'étudiants mais aussi par les travaux des traducteurs chevronnés, on restera en face de différents types d'écarts relevant de mécanismes fort diversifiés. Deux cas extrêmes se présentent a priori: l'appauvrissement de la traduction par rapport à l'original ou son contraire - l'enrichissement. Entre ces deux pôles, on trouve une multitude des cas attestés aussi bien dans les traductions des oeuvres littéraires que dans celles de textes techniques, d'articles de presse, et autres.

Commençons par jeter un coup d'oeil rapide sur les deux cas extrêmes.

${ }^{3}$ Pour une revue des types d'équivalence on consultera aussi avec profit A. Pisarska, T. Tomaszkiewicz (1996) 


\subsection{FORUM OU L'EXIGENCE DE LECTURE RAPIDE ET AISÉE}

L'omission pure et simple d'éléments divers de l'original est attestée souvent dans les textes destinés au grand public. L'exemple classique est celui de l'hebdomadaire polonais Forum qui reproduit en version polonaise un choix d'articles tirés de la presse mondiale. La suppression d'éléments divers y est de règle, à un point tel que bien rares sont les textes reproduisant intégralement le contenu de l'original. Les omissions relevées obéissent dans $90 \%$ des cas à une règle qu'on pourrait baptiser l'exigence de lecture rapide et aisée. Ainsi le texte traduit ne doit jamais poser la moindre difficulté au lecteur qui a tendence à négliger les notes explicatives l'informant des détails de la réalité familiers aux lecteurs de l'original mais inconnus des lecteurs de la traduction. Chaque fois que la compréhension du message global n'est pas compromise, sont systématiquement éliminées toutes sortes d'allusions trop dépendantes de tout contexte culturel, linguistique ou autre pour peu qu'elles soient illisibles pour le lecteur polonais ${ }^{4}$.

\subsection{L'AMPLIFICATION OU LE SOUCI DE L'EXPLICITE}

A l'opposé on trouve l'amplification - un procédé non moins répandu qui consiste à ajouter des éléments non-nécessaires dans la traduction. Une thèse récente ${ }^{5}$ a permis d'examiner en détail les mécanismes de ce procédé très fréquent - qu'ils soient conditionnés par le système linguistique que ceux relèvent entièrement des choix individuelles. Trois exemples suffisent:

(1) Qui ... retranchait l'inutile (XII, 20) - (Nie folgujac) zbytecznym gatazkom obcinat je (K)

(2) (Il) mettra à sec la mare et tous ses habitants (VI, 12) - Wysuszq staw i wód mieszkanki, żaby $i$ kijanki (S)

(3) Il prescrit à ses amis un universel abatis (XII, 20) - Wmówił sqsiadom...rzeź odrośli i gałazek $(\mathrm{K})$

Le contenu de l'adjectif inutile est rendu par zbyteczny, le substantif gałazki 'branches', ajouté par le traducteur, n'est pas contenu directement dans l'original. De même, żaby i kijanki 'grenouilles et têtards' amplifie, de façon fort heureuse d'ailleurs, le substantif habitants rendu par mieszkanki. Dans (3) enfin, on chercherait en vain odrośle (odrośli) et gatazka (gatazek) dans le texte original.

\footnotetext{
${ }^{4}$ Ces remarques sont tirées de la comparaison d'une cinquantaine d'articles parus dans Forum au cours de l'année 1992 avec leur version originale.

${ }^{5}$ Cf. M. Szeflińska-Karkowska, L'amplification dans les traductions des textes du français en polonais. Thèse de doctorat, Łódź 1998.
} 


\section{LES TYPES D'ÉCARTS DANS LES TRADUCTIONS DES FABLES DE LA FONTAINE}

Ces exemples viennent de notre corpus qui contient des traductions des Fables de La Fontaine effectuées par des poètes dont ni la connaissance du français ni la compétence linguistique en polonais ni enfin le savoir-faire poétique ne peuvent être mis en doute. Nous en avons donc comparé deux versions polonaises; elles étaient élaborées par Leopold Staff, Stanisław Komar et Julian Rogoziński ${ }^{6}$.

Ce qui frappe à la lecture de notre corpus, c'est la variété et le nombre d'écarts 7 . Ceux-ci existent entre l'original et les versions polonaises d'un côté mais aussi entre deux traductions polonaises. Ainsi entre les textes polonais suivants, les oppositions concernent le choix de registres de la langue:

$$
\begin{aligned}
& \text { J'inspirais ici l'amour (XI, 4) - Zbudzić w was chcę ukochanie }(S)-\text { Sen } \\
& \text { ten miłość oznacza }(\mathrm{K})
\end{aligned}
$$

Suivre une plus douce vie (XII, 20) - Prowadzić mite i przyjemne życie (S) - Jaśniejszego, stodszego żywota zaznać (K)

Il exprima ... le caractère de l'idole (IX, 6) - Zdziałat konterfekt Jowiszowy (R) - Wykut... postać Jowiszowa (K)

Elle offre à ses amants des biens (XI, 4) - Daje ona kochankom swym (S) ... która użycza swoim kochankom $(\mathrm{K})$

Tout meurt (XII, 20) - Wszystko kona (S) - Wszystko zmarniato (K) Chacun ... crut que (VI, 12) - Ludziska ... zbiegli się myślac, ze ... (S) Gapie, .... mniemali, ze ... (K)

Le cas de La Fontaine, écrivain du XVII ${ }^{\mathrm{e}}$ siècle, a ceci de particulier que ses fables donnent lieu à la possibilité - absente chez les auteurs contemporains - de recourir à l'archaïsation lors de la traduction. Notre corpus présente de nombreuses formes sorties d'usage de nos jours. Elles concernent le niveau lexical, sémantique, et formel (flexionnel, morphologique et dérivationnel).

${ }^{6}$ Cf. J. de La Fontaine, Bajki, tł. S. Komar, Ossolineum, Wrocław 1954. Les traductions de L. Staff et de J. Rogoziński ont été publiées dans J. de La Fontaine, Bajki, tt. J. Dackiewicz i in. PIW, Warszawa 1986.

Dans les exemples que nous citons dans la suite de cet article, les sigles (K), (R) et (S) renvoient, respectivement à S. Komar, à J. Rogoziński et à L. Staff.

Les exemples cités (leur relevé complet se trouve dans le mémoire de maîtrise préparé à l'Université de Varsovie par M. Wieliczko 1998) illustrent parfois plus d'un point. Ainsi la traduction de (i) $l l$ conseille à ses voisins (XII, 20) par (ii) Squsiadom wmówił (K) s'écarte de l'original sur le plan lexical et morphologique (d'un autre côté, on trouve sous la plume de L. Staff: radzi swym sqsiadom): conseiller correspond à wmawiać/wmówić 'persuader', en face du présent de l'indicatif de l'original on trouve le prétérit polonais (wmówil et non wmawia).

${ }^{7}$ En partie ils sont dus aux exigences formelles de la poésie: rythme, rime etc. 


\subsection{ARCHAÏSMES LEXICAUX}

Considérons:

(10) Je lui voue ... de nouveaux sacrifices $(\mathrm{XI}, 4)$ - Na cześć mu ztozyć ... nowe obiaty (K)

(11) . On sait assez que .... le Destin adresse... (VI, 18) - Snać to los biesi wiedzie tam... (K)

(12) Le Soleil eut dessein...de songer à l'hyménée (VI, 12) - Stońce miato ochotę... zagiew hymenu zapalić (K)

Le mot obiaty employé dans (10) est sorti d'usage, de même que l'adjectif biesi attesté dans (11). L'exemple (12) enfin présente hymen traduisant hyménée employé dans le texte français - tous les deux archaïques.

\subsection{ARCHAÏSMES SÉMANTIQUES}

Dans

(13) Tremblez, humains, faites des voeux (IX, 6) - Drziyjie śmiertelnicy, duchem znosicie wota $(\mathrm{R})$

le mot duchem est archaique dans l'emploi adverbial.

\subsection{ARCHAÏSMES FORMELS (FLEXIONNELS, MORPHOLOGIQUES, DÉRIVATIONNELS)}

\section{Considérons:}

(14) Les divers mouvements inconnus à nos yeux (XI, 4) - Obroty niedostepne dla ócz naszych sięgu (K)

(15) Ce n'étaient que voeux et qu'offrandes (IV, 8) - Ciagle śluby i prezenta (R)

(16) Il n'en dut guère des dieux (IX, 6) - Podobnym byt tu do poety, który gdy zmyślit bogi $(\mathrm{R})$ - Nie inny byt od rzézbiarza poeta, co zmyślit bogi $(\mathrm{K})$

(17) Qu'il ait en sa main un tonnerre (IX, 6) - Niech mu piorun z rak wylata (K)

Le caractère archaique est visible dans les mots: ócz (au lieu de oczu), prezenta (au lieu de prezenty), bogi (à la place de bogowie) et wylata (pour wylatuje).

Dans

(18) L'homme est de glace aux vérités (IX, 6) - Człek dla prawdy jak lód zgota (K) (19) Il eut au fond de l'ermitage ... (VII, 3) - W pośrodku sera miat schron (K)

le caractère archaïque est lié à l'emploi de deux formes: człek (au lieu de czlowiek) et $\boldsymbol{w}$ pośrodku (au lieu de pośrodku).

Mettons de côté cet aspect, somme toute stylistique, pour passer à l'analyse sémique. 


\subsection{ANALYSE SÉMIQUE}

La question qu'on peut se poser consiste à se demander si les traits sémantiques des mots de l'original sont les mêmes que ceux des traductions. Si on néglige le contenu affectif des signifiés, on relève, a priori, quatre types de relations: identité, inclusion, intersection et disjonction. Toutes les quatre sont attestées dans notre corpus.

\subsubsection{L'IDENTITÉ}

Cette relation équivaut, purement et simplement, à la fidélité à l'original:

(20) Elle accoucha d'une souris (V, 10) - Urodzita mysz (S) - Powita mysz (K)

(21) Quittez-moi votre serpe (XII, 20) - Oddaj ten nóż (S)

(22) Aux noces d'un tyran (VI, 12) - Podczas weselnych godów tyrana (S) - W weselne gody tyrana $(\mathrm{K})$

(23) ...dans un fromage de Hollande (VII, 3) - (Osiadl) w serze holenderskim (R) - W holenderskim serze pustelnicze obrat leze (K)

(24) Mon char marche à souhait (VI, 18) - Wóz jedzie gtadko (S) - Wóz jedzie $(\mathrm{K})$

(25) Le dormeur s'éveilla (XI, 4) - Gdy śpiacy się obudzit (S,K)

(26) Tout le peuple ... noyait son souci (VI, 12) - Lud ... topit swe troski (S) Lud topil swe biedy (K)

\subsubsection{L'INCLUSION}

L'inclusion des traits sémantiques d'un terme dans l'ensemble des sèmes de l'autre se retrouve dans la relation d'hyponymie/hypéronymie:

(27) Celui-ci retranche de l'âme désirs et passions - I ten obcina w duszy żadze i pragnienia $(\mathrm{S})$ - I ten obcina ... co z duszy wynika: pragnienia, namiętności (K)

(28) Je trouve une douceur secrète (XI, 4) - Kryjesz uroki tajemnicze (S)

(29) Qui m'arrêtera sous vos sombres asiles (XI, 4) - Któz mi zapewni ustroń błogq (S) - Któz mi zapewni wasze ustronie zaciszne (K)

(30) Les divers mouvements inconnus à nos yeux (XI, 4) - Obroty niedostepne dla ócz naszych sięu (K)

(30) Je me figure un auteur $(\mathrm{V}, 10)$ - Przypomina poete (S)

(31) On oult d'une commune voix ... des citoyennes des étangs ... (VI, 12) - Ustyszano jak wielkim rechotem ...(K)

(32) Il vit en certains lieux un sage (XII, 20) - Spotkat w mieścinie matej ... (S) - Spotkat w ich kraju ...(K) 


\subsubsection{L'INTERSECTION}

L'intersection de deux signifiés se présente lorsque ceux-ci ne partagent qu'une partie de sèmes:

(33) Contre de telles gens ... je réclame (XII, 20) - Dla takich kpów mam tylko stowa potępienia $(\mathrm{K})$

(34) Qui touchait ... même les malheureux (XI, 4) - ... i nędzarza mógt dotknać (K)

(35) Que si je ne suis né pour de si grands projets (XI, 4) - Jeślim niegodny tych wielkich zadań $(\mathrm{K})$

(36) ... dont les travaux sont si célèbres (VI, 18) - Które czyny swymi szeroko stynie $(\mathrm{K})$

(37) ...et m'apprendre des Cieux (XI, 4) - Bym...na niebie badat (S)

(38) Je chanterai la guerre que... $(\mathrm{V}, 10)-W$ tym ujrze i opisze boje (S) - I wyśpiewam bój ... (K)

\subsubsection{LA DISJONCTION}

On a affaire à la disjonction lorsqu'on ne trouve aucun sème commun entre les deux ensembles des traits sémantiques correspondant à deux unités lexicales.

(39) Son bonheur consistait aux beautés d'un jardin (XII, 20)-Swe cate szczęście widzial w piękności ogrodu $(\mathrm{S})$

(40) Le dormeur s'éveilla, tant il en fut surpris (XI, 4) - Gdy spiacy się obudzit, dtugo sen rozważa $(\mathrm{K})$

(41) Il était loin de tout humain secours (VI,18) - Z dala od siedzib ludzkich (K)

(42) Un philosophe austère, et né dans la Scythie... voyagea chez les Grecs (XII, 20) - Chtop scytyjski, filozof jak prosty kót z ptotu ... powędrowat do Greków (K)

Il est clair que pour celui qui s'intéresse au degré de fídélité de la traduction à l'original, seules l'intersection et l'inclusion sont pertinentes. L'identité sémique équivaut à la fidélité totale, la disjonction sémique est son contraire.

\subsection{LES INCONVÉNIENTS DE L'ANALYSE SÉMIQUE}

L'inconvénient qui est lié à l'analyse sémique tient cependant au fait que pour pouvoir évaluer l'identité ou le degré d'écart entre l'original et sa traduction, il est impératif de disposer de données précises concernant la composition sémique des mots du texte (ou, si l'on raisonne en termes de représentations sémantiques, celles relatives à la structure de celles-ci). Différentes analyses sémiques ont été faites, à commencer par celle, devenue célèbre du mot siège et de son champ sémantique (cf. 
Pottier 1964), celles de Katz et Fodor (1966-1967) et d'autres ${ }^{8}$. Elles ne visent cependant qu'un secteur extrêmement limité du lexique. En plus, à quelques exceptions près, elles se cantonnent dans le domaine nominal et restent peu fiables. Dans ces conditions, en partant de ces données limitées, la distance à parcourir est grande avant d'arriver à calculer la composition sémique de la totalité du texte.

D'un autre côté, on sait très bien que la configuration sémique - ou la représentation sémantique d'un texte - n'est qu'une partie de ce qu'il convient de traduire: d'autres aspects (pour ne citer que le côté pragmatique, connotatif, esthétique etc.) s'y greffent et demandent à être traduits pour que le texte final soit déclaré fidèle à l'original'.

Il serait erroné cependant de croire que plus grand est le nombre d'écarts, plus on s'expose au danger de déformer le message original au point de mériter le label de traduction infidèle, ou d'erreur de traduction. Les exemples que nous avons cités jusqu'à présent restent tous dans les limites de l'acceptable: on ne peut pas parler ni de traduction infidèle ni d'erreur. Toutefois si par exemple l'on compare la composition sémique de lieux d'un côté et celle de mieścina 'petite ville' ou kraj 'pays' de l'exemple (32), on est amené à conclure que la différence concerne plus d'un sème. Il en est de même dans (2) (Il) mettra à sec la mare et tous ses habitants (VI, 12) Wysusza staw i wód mieszkanki, żaby i kijanki (S) et dans beaucoup d'autres exemples. De façon générale, les traductions des Fables de La Fontaine que nous avons examinées abondent en exemples illustrant les relations d'inclusion, d'intersection et même de disjonction citées aux points 3.3.2 - 3.3.4. Leur lecture est cependant loin de donner l'impression globale de traduction infidèle ou erronnée. Il semble donc que ce qui compte dans l'évaluation finale c'est la réponse à la question si les écarts sur le plan sémique ont été compensés à d'autres niveaux: pragmatique, affectif, esthétique etc. On sera prêt à pardonner au traducteur d'employer dans tel contexte spécifique un hypéronyme à la place d'un synonyme exact ou de recourir à l'amplification à condition, par exemple, de garder intact le message général, de restaurer les réseaux associatifs des termes de l'oiriginal etc.

Il existe cependant des contextes critiques dans lesquels le retranchement (ou l'addition) d'un seul sème conduit à une déformation radicale du message de l'original et peut provoquer des conséquences graves sur le plan pragmatique. Tel est le cas de

\section{Ceci est un rectangle - To jest kwadrat}

On y trouve rectangle rendu par $\boldsymbol{k w a d r a t}$ 'carré'. Il est clair que la seule différence entre les deux termes concerne un sème unique: [+ à côté égaux] attesté dans $k w a d r a t$ 'carré' et absent de rectangle.

\footnotetext{
${ }^{8}$ Les représentations sémantiques semblent plus nombreuses (on consultera avec profit Mel'cuk 1984-1993 pour un type de description et sa bibliographie pour des propositions concurrentes). Elles sont faites selon différentes techniques et utilisent des outils qui ne sont pas manifestement compatibles les uns avec les autres.

${ }^{9}$ Cf. d'un côté Grucza (1981, 1985, 1986), Mounin (1963), Langage (1982), Pieńkos (1993) et de l'autre Vinay, Darbelnet (1971), Wojtasiewicz (1966).
} 
Donnée comme réponse à la question

Comment appelle-t-on une figure géométrique plane ayant quatre côtés de longueur égale et quatre angles droits?

la réponse (43), correcte dans l'original, devient incorrecte dans la traduction et ne saurait donc être acceptée.

\subsection{LA TRADUCTION ET LA MORPHOSYNTAXE}

Sur un autre plan, on peut classer les manquements au principe de fidélité compte tenu de la structure morphosyntaxique des deux langues. Nous n'insisterons pas longtemps sur l'incompatibilité des moyens du système morphosyntaxique dont disposent la langue-source et la langue-cible. Cette incommensurabilité vient de l'absence de certaines formes morphologiques ou flexionnelles et de certaines structures syntaxiques en français et en polonais. Tel est par exemple le cas d'articles: définis, indéfinis, contractés, partitifs inexistants en polonais. Dans le domaine verbal, en ce qui concerne le temps et plus particulièrement l'aspect (la différence ne s'y exprime pas en termes de présence/absence de formes), la divergence systémique entre les deux langues est d'un autre type mais non moins sensible. Peut-on parler dans de tels cas d'infidélité de la traduction à l'original si on n'a pas d'équivalence terme à terme entre les deux langues? Comment traiter les très nombreux exemples portant sur les éléments du système verbal? La question reste ouverte. Ce que nous soulignerons ici, c'est que parfois la façon de traduire ces catégories manquantes s'écarte de ce qu'on en dit dans les études confrontatives du français et du polonais ${ }^{10}$. Ainsi pour l'article partitif l'on trouve:

(45) Votre songe a du sens (XI, 4) - Ma on swój sens (K)

(46) Dans ce songe...soupçonnant du mystère (XI, 4) - Sqadzac, że tajemnica jakaś się w tym kryta (S)

avec un possessif ou un indéfini polonais à la place d'un partitif en français.

En ce qui concerne l'article défini on trouve assez souvent un possessif:

(47) Je vous rends mille grâces, les Betles (I, 17) - Moje lube... z wdzięczności się caty rozptywam $(\mathrm{R})$ - Stokrotne dzięki, moje piękne panie $(\mathrm{K})$

(48) L'homme en avait sa part (IV, 8) - Dotknęto to naszego czteka $(\mathrm{R})$

(49) L'artisan exprima si bien... (IX, 6) - I zdziatat majster nasz tak celnie (R)

mais parfois rien du tout:

(50) Au dévot personnage des députés... s'en vinrent (VII, 3) - Ów bigot gruby...delegacje wita $(\mathrm{R})$ - Do nabożnej tej persony przyszli delegaci $(\mathrm{K})$

\footnotetext{
${ }^{10}$ Cf. S. Gniadek (1979).
} 
Lorsqu'on examine les secteurs du système grammatical qui se correspondent simplement et où à une catégorie dans la langue de l'original correspond le même type de catégorie dans la langue-cible, on relève à côté de la fidélité absolue des cas d'écarts notables. Voici quelques exemples:

- le pluriel morphologique est rendu par un collectif ou inversement ce qui est compréhensible vu la parenté sémantique évidente entre les deux formes:

(51) La guerre que firent les Titans au maître du tonnerre (V, 10) - Bój,... który zwiódł Tytaniczny ród $z$ władcq piorunów... (K)

(52) Ils alloient en terre étrangère (VII, 3) - Musieli ruszyć w obce strony (R)

- la forme morphologique du présent de l'indicatif (avec différentes valeurs sémantiques) est rendue par celle du passé, du futur, du conditionnel, même - ce qui est surprenant - par l'impératif etc.:

(53) Ils alloient... chercher quelque secours (VII, 3) - Positków do obrony szukaja $w$ podrózy $(\mathrm{K})$

(54) Ceux qui font voeux d'être siens (VII, 3) - Temu, kto złoży im zakonne śluby (R) - Tym co z siebie ztożyli ofiarę $i$ śluby $(\mathrm{K})$

(55) Une voix qui lui parle ainsi (VI, 18) - Gtos, który tak mówi (S) - Gtos tak przemówit $(\mathrm{K})$

(56) Esope... trouvait que (VI, 12) - Ezop... wyciagnal wnioski (S) - Jak Ezop powiada (K)

(57) Il invoque à la fin le dieu $(\mathrm{VI}, 18)$ - Wreszcie jal wzywać bóstwo $(\mathrm{K})$ - Wreszcie przyzywa bóstwo (S)

(58) Le secours serait prêt dans quatre ou cinq jours (VII, 3) - Positki nadciagna za jakich dni parę $(\mathrm{R})$

(59) Ton bras peut me tirer (VI, 18) - Wybaw mnie! (S) - Wyrwij mnie! (K)

- en ce qui concerne l'adjectif démonstratif, on relève tantôt des cas de fidélité grammaticale absolue comme dans

(60) Le Scythe alors lui demanda: Pourquoi cette ruine? (XII, 20) - Scyta rzecze: Na cóz to spustoszenie? (K) - Scyta pyta: Po co takie zniszczenie? (S)

(61) Romps ce caillou qui te nuit (VI, 18) - Pottucz ten kamień spod kota (S)

tantôt on oublie de traduire l'adjectif démonstratif:

(62) Rozkrusz kamienie, aby kót nie uwieraty (K)

- le même cas se présente avec l'adjectif qualificatif: un indéfini à la place d'un adjectif qualificatif comme dans (63) ou des adjectifs qualificatifs en polonais comme en français:

(63) Il vit en certains lieux un sage (XII, 20) - W mieścinie małej spotkal mędrca (S)

(64) Ote d'autour de chaque roue ce malheureux mortier (VI, 18) - Oczyść kota z tej paskudnej gliny (S) - Oczyść koła z błotnej mazi (K). 


\section{CONCLUSION}

Il est temps de conclure.

Les écarts sont le pain quotidien du traducteur. Parfois, comme dans notre corpus, elles sont justifiées en partie par les exigences formelles de la poésie: rythme, rime etc. Certaines libertés que prennent les traducteurs sont fondées sur les affinités manifestes: ainsi le subjonctif dont l'une des valeurs est de transmettre un ordre est rendu par l'impératif, le pluriel correspond au collectif etc.

Toute tentative de quantification de l'écart nous paraît cependant risqué. En effet, un calcul opéré sur les sèmes ou une comparaison de représentations sémantiques ne rendrait compte que d'un aspect parmi d'autres de deux textes et ne saurait fournir une évaluation fiable et définitive d'éventuels écarts séparant un original de sa traduction. Vouloir affiner les critères de comparaison et surtout chercher à les quantifier passe nécessairement par une évaluation des connotations des mots, de leurs valeurs affectives, stylistiques, esthétiques et autres facteurs qui pour le moment échappent à la quantification.

\section{BIBLIOGRAPHIE}

Catford, J. C. (1965), A Linguistic Theory of Translation, Oxford University Press, London.

Gniadek S. (1979), Grammaire contrastive franco-polonaise, Państwowe Wydawnictwo Naukowe, Warszawa.

Grucza, F. (1981), Glottodydaktyka a translatoryka. Materialy z IV Sympozjum Instytutu Lingwistyki Stosowanej Uniwersytetu Warszawskiego, Warszawa.

Grucza, F. (1985), Lingwistyka, glottodydaktyka, translatoryka. Materiały z VIII Sympozjum Instytutu Lingwistyki Stosowanej Uniwersytetu Warszawskiego, Wydawnictwa Uniwerytetu Warszawskiego, Warszawa.

Grucza, F. (1986), Problemy translatoryki i dydaktyki translatorycznej. Materiały Sympozjum Instytutu Lingwistyki Stosowanej Uniwersytetu Warszawskiego, Wydawnictwa Uniwerytetu Warszawskiego, Warszawa.

Hurtado-Albir A. (1990), La notion de fidélité en traduction, Didier Erudition, Paris 1990.

Katz J. J., J. A. Fodor (1966-1967), Structure d'une théorie sémantique, Cahiers de Lexicologie. Koller W. (1979), Einführung in die Übersetzungwissenschaft, Queller \& Meyer, Heidelberg.

La Fontaine J. (1954), Bajki, tł. S, Komar, Ossolineum, Wrocław.

La Fontaine J. (1986), Bajki, tł. J. Dackiewicz i in., PIW, Warszawa.

La Fontaine J. (1960), Fables, Garnier Frères, Paris.

Langage (1982), J.-R. Ladmiral éd., La traduction, $\mathrm{n}^{\circ} 28$.

Ljudskanov, A. (1969) Traduction humaine et traduction mécanique, Dunod, Paris.

Łukszyn J. (1993), Tezaurus terminologii translatorycznej, PWN, Warszawa.

Mel'cuk I. (1984-1993), Dictionnaire explicatif et combinatoire du français contemporain. Recherches lexico-sémantiques, Les Presses de l'Université de Montréal. Montréal.

Mounin, G. (1963), Les problèmes théoriques de la traduction, Gallimard, Paris.

Nida, E. (1964), Towards the Science of Translation, Brill, Leiden. 
Nid a, E. (1975), Language Structure and Translation, University Press, Stanford, California.

Pi eńk os, J. (1993), Przektad i tlumacz we wspótczesnym świecie, Wydawnictwo Naukowe PWN, Warszawa.

Pisarska A., Tomaszkiewicz T. (1996), Wspótczesne tendencje przektadoznawcze, Wydawnictwo Naukowe UAM, Poznań.

Pottier, B. (1964), Vers une sémantique moderne, Travaux de Linguistique et de Littérature, II-1, pp. 107-137.

Seleskovitch, D., Lederer, M. (1986), Interpréter pour traduire. Publications de la Sorbonne, Collection Traductologie 1, Didier Erudition, Paris.

Sk oru pka, S. (1996), Stownik frazeologiczny jezyka polskiego, Wiedza Powszechna, Warszawa. Szefli íska-Karkowska M. (1998), Le procédé d'amplification dans la traduction des textes du français en polonais. Thèse de doctorat, Université de Łódź.

V in a y, J. P., Da rbel net, J. (1971), Stylistique comparée du français et de l'anglais, Didier, Paris. Wi eliczko M. (1998), Les traduction des «Fables» de La Fontaine en polonais - divergences et identités. Mémoire de matrise, Université de Varsovie.

Wojtasiewicz, O. A. (1996), Wstęp do teorii ttumaczenia. Polskie Towarzystwo Tłumaczy Ekonomicznych Prawniczych i Sadowych TEPIS, Warszawa. 